\title{
Functional insights of nucleocytoplasmic transport in plants
}

\author{
Kentaro Tamura and Ikuko Hara-Nishimura* \\ Department of Botany, Graduate School of Science, Kyoto University, Kyoto, Japan
}

\section{Edited by: \\ Katja Graumann, Oxford Brookes \\ University, UK}

\section{Reviewed by:}

Dana Reid MacGregor, University of Exeter, UK

Geraint Parry, University of Liverpool, UK

\section{${ }^{*}$ Correspondence:}

Ikuko Hara-Nishimura, Department of Botany, Graduate School of Science, Kyoto University, Kyoto 606-8502, Japan

e-mail: ihnishi@gr.bot.kyoto-u.ac.jp
Plant nucleocytoplasmic transport beyond the nuclear envelope is important not only for basic cellular functions but also for growth, development, hormonal signaling, and responses to environmental stimuli. Key components of this transport system include nuclear transport receptors and nucleoporins. The functional and physical interactions between receptors and the nuclear pore in the nuclear membrane are indispensable for nucleocytoplasmic transport. Recently, several groups have reported various plant mutants that are deficient in factors involved in nucleocytoplasmic transport. Here, we summarize the current state of knowledge about nucleocytoplasmic transport in plants, and we review the plant-specific regulation and roles of this process in plants.

\section{Keywords: nucleocytoplasmic transport, nuclear transport receptors, nucleoporins, nuclear pore complex Arabidopsis thaliana}

\section{INTRODUCTION}

The nucleus is the most prominent organelle in eukaryotes and is surrounded by the nuclear envelope, which provides a controlled barrier between the nucleoplasm and the cytoplasm. The nuclear envelope consists of a double membrane spanned by nuclear pore complexes (NPCs), which form channels, allowing the passive diffusion of small molecules. Macromolecules larger than $\sim 40 \mathrm{kDa}$ are transported actively across the nuclear envelope through the NPC in a regulated manner (Grossman et al., 2012; Raices and D'Angelo, 2012). How the rapid, bidirectional trafficking of thousands of specific cargoes through the NPC is achieved has been the subject of intense study (Mosammaparast and Pemberton, 2004). In addition to bulk transport of constitutive nuclear proteins, changes in gene expression generally require the controlled import/export of key signaling molecules to/from the nucleus (Stewart, 2007; Merkle, 2011), indicating that nucleocytoplasmic transport is a highly dynamic process.

The first step in nucleocytoplasmic transport is the recognition of cargo molecules by specific transport receptors, including importins and exportins (Bednenko et al., 2003; Mosammaparast and Pemberton, 2004; Stewart, 2007). Following formation of the cargo-transport receptor complex, the NPC mediates translocation of this complex through the nuclear envelope. Such selective transport of molecules across the nuclear envelope plays an important role not only in basic cellular activity, but also in cellular differentiation and responses to environmental signals (Raices and D’Angelo, 2012; Parry, 2013). Recent investigations of nucleocytoplasmic transport have revealed unique roles and unexpected layers of regulation of this process in plants (Meier and Somers, 2011). In this review, we summarize studies involving mutants defective in nucleocytoplasmic transport factors, and we discuss the key roles of the nucleocytoplasmic transport system in plant cells.

\section{IMPORTIN $\beta$ FAMILY IN Arabidopsis thaliana}

Importin $\beta$ (or karyopherin $\beta$ ) family proteins are major nuclear transport receptors that interact with Ran small GTPase and mediate the nuclear transport of specific cargoes (Lott and Cingolani, 2011; Merkle, 2011). These proteins are also referred to as importins or exportins depending on whether they mediate cargo import into, or export out of, the nucleus. The structures of importin $\beta$ proteins are characterized by a similar series of helical HEAT repeats, which are approximately 40 residues in length (Strom and Weis, 2001; Xu et al., 2010; Lott and Cingolani, 2011). The fundamental repeat unit is a right-handed superhelical structure consisting of a hairpin comprising two $\beta$ helices. Each hairpin is connected to the next by a linker region. Crystal structural analysis has indicated that mammalian importin $\beta$ is composed of 19 HEAT repeats and uses extensive interaction interfaces to associate with different cargoes (Cingolani et al., 1999, 2002; Lee et al., 2003).

The Arabidopsis thaliana genome encodes 18 importin $\beta$ proteins (Figure 1), while the Saccharomyces cerevisiae genome encodes 14 importin $\beta$ proteins and the Homo sapiens genome encodes more than 20 of these proteins (Goldfarb et al., 2004). At least 16 subfamilies of importin $\beta$, each containing representatives from eukaryotic subgroups, have been identified (Figure 1), suggesting that these importin $\beta$ subfamilies were established prior to eukaryotic radiation (O'Reilly et al., 2011). Plant genomes lack the exportin 6 (XPO6) gene family, while embryophytespecific sequences have been identified, which were designated PLANTKAP (At3g17340 in A. thaliana; O'Reilly et al., 2011; Figure 1).

\section{IMPORTIN $\alpha$ FAMILY IN A. thaliana}

The best understood function of importin $\alpha$ is to serve as an adaptor that links classical nuclear localization signal (cNLS)containing proteins to importin $\beta$, which interacts with the ternary complex at the NPC. Importin $\alpha$ comprises two functionally and structurally distinct domains, namely, the flexible $\mathrm{N}$-terminal importin $\beta$-binding (IBB) domain and a C-terminal domain that consists of eight to nine tandem armadillo (ARM) repeats (Goldfarb et al., 2004; Marfori et al., 2011). The stacking of helical ARM repeats generates a right-handed superhelical structure, forming 


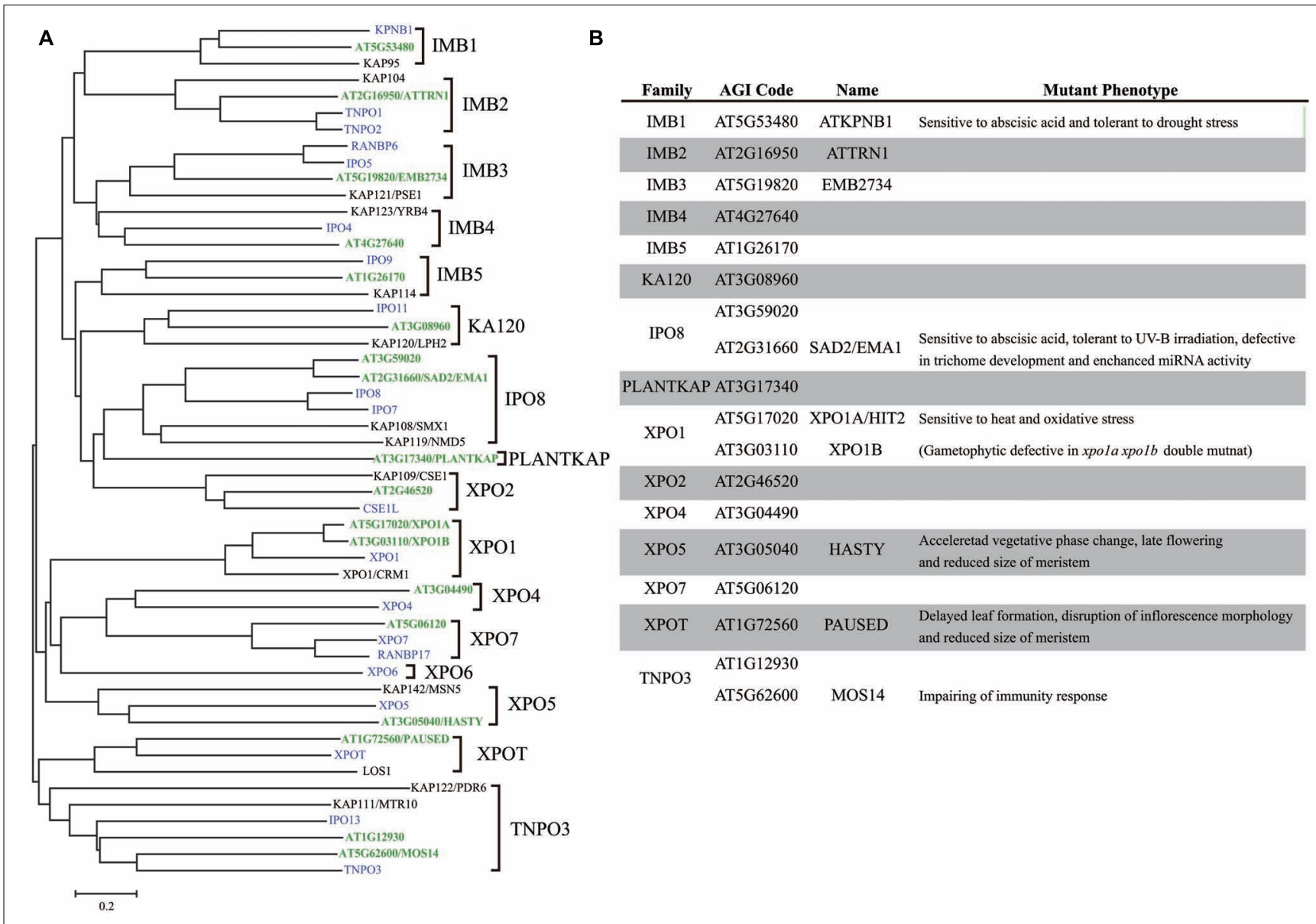

FIGURE 1 | Comparison of importin $\beta$ family proteins in Arabidopsis, human, and yeast. (A) Phylogenetic tree of importin $\beta$ family in Arabidopsis (green), human (blue), and yeast (black) constructed using the neighbor joining method. (B) Arabidopsis importin $\beta$ family proteins.

two separate cNLS-binding sites (the major and minor sites; Conti et al., 1998; Marfori et al., 2011). Chang et al. (2012) determined the crystal structure of rice importin $\alpha 1 \mathrm{la}$ at $2-\AA \AA$ resolution. Consistent with S. cerevisiae and H. sapiens proteins, Oryza sativa importin $\alpha$ preferentially binds to the prototypical cNLS from SV40 large T-antigen at the major nuclear localization signal (NLS) binding site. On the contrary, two plant-specific NLSs (Kosugi et al., 2009) bind to the minor site of rice importin $\alpha$. Interestingly, mouse importin $\alpha$ binds to these plant-specific NLSs at the major site, suggesting that plant importin $\alpha$ has plant-specific features to mediate nuclear import.

The number of importin $\alpha$ family genes has increased over the course of evolution; yeast has a single importin $\alpha$ gene, Drosophila melanogaster has four genes, and $H$. sapiens has six genes (Mason et al., 2009; Yasuhara et al., 2009; Figure 2). H. sapiens, D. melanogaster, and most animal importin $\alpha$ genes fall into one of three conserved clades, i.e., $\alpha 1, \alpha 2$, and $\alpha 3$ (Mason et al., 2002 , 2009). By contrast, plant and fungal importin $\alpha$ belong to $\alpha 1$ and a non-conventional family (Figure 2), suggesting that the importin $\alpha 1$ gene may be the earliest progenitor of importin $\alpha$, and $\alpha 2$ and $\alpha 3$ are metazoan specific importin $\alpha$. A. thaliana has eight importin $\alpha$ proteins (IMPA1-7 and 9) that contain both
IBB domains and ARM repeats and a single protein (IMPA8) that contains only ARM repeats. Based on sequence similarity, A. thaliana IMPA9 is likely to be a non-conventional importin $\alpha$ that does not fall into the $\alpha 1, \alpha 2$, or $\alpha 3$ groups (Figure 2). A. thaliana IMPA1 binds to all three types of NLS in vitro, which were identified in maize and SV40 (Smith et al., 1997). Moreover, IMPA1-4 interact with $A$. thaliana CAS (cellular apoptosis susceptibility), which is required for recycling importin $\alpha$ from the nucleus to the cytosol, indicating that nucleocytoplasmic transport is functionally conserved in plants (Haasen and Merkle, 2002).

\section{STRUCTURE OF THE NUCLEAR PORE COMPLEX}

The NPC is a macromolecular assembly of protein subcomplexes that serves as a key regulator of molecular trafficking between the cytoplasm and the nucleus (Grossman et al., 2012; Parry, 2013; Tamura and Hara-Nishimura, 2013). Extensive studies have identified more than 30 different nucleoporin proteins that serve as building blocks of the NPC (Rout et al., 2000; Cronshaw et al., 2002; Tamura et al., 2010). By contrast to other nuclear envelope proteins, NPC components are evolutionarily conserved between yeast, animals, and plants, indicating that these NPCs share a common progenitor (Devos et al., 2006). Based on their function 


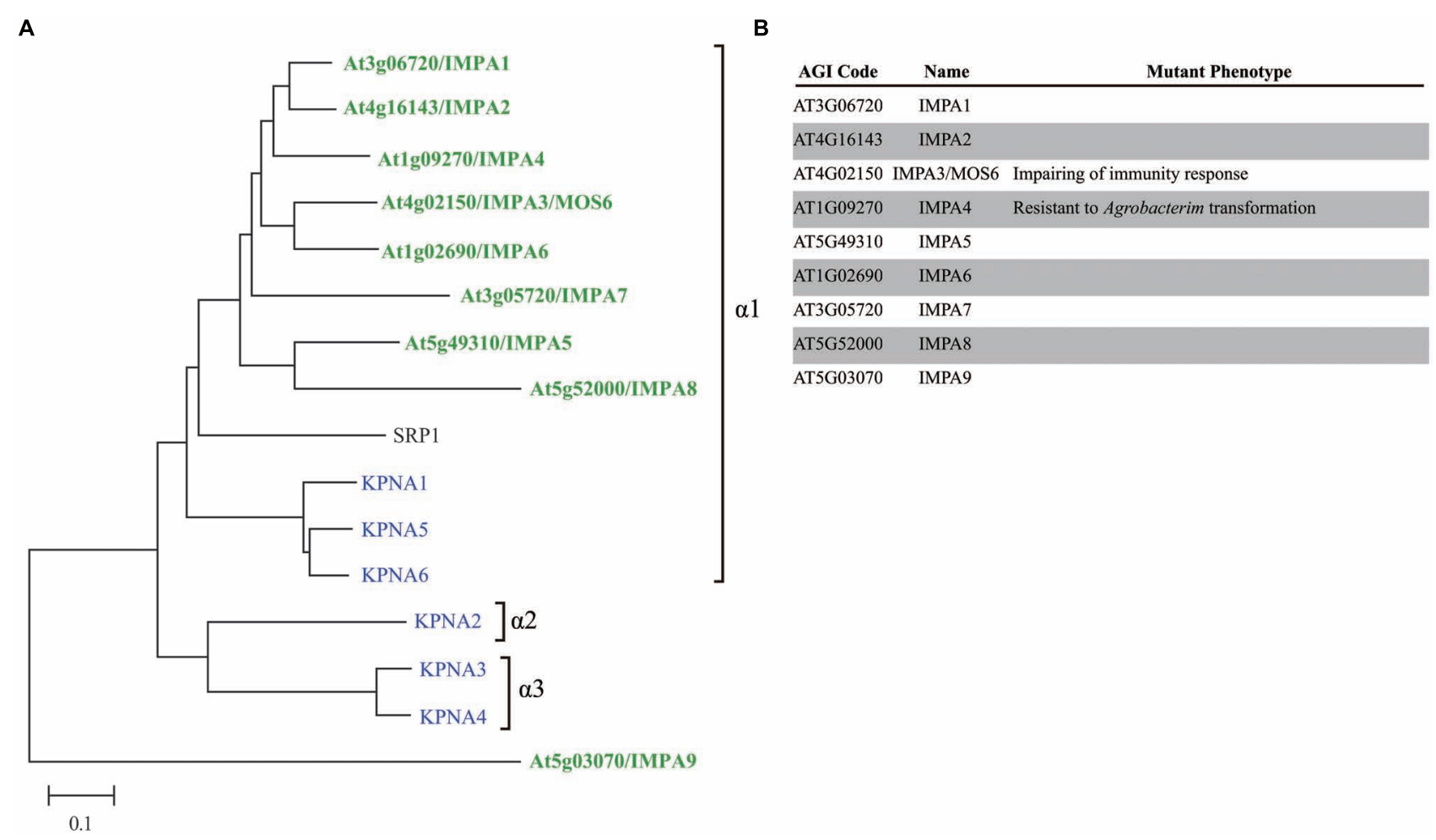

FIGURE 2 | Comparison of importin $\alpha$ family proteins in Arabidopsis, human, and yeast. (A) Phylogenetic tree of importin $\alpha$ family in Arabidopsis (green), human (blue), and yeast (black) constructed using the neighbor joining method. (B) Arabidopsis importin $\alpha$ family proteins.

in the NPC, nucleoporins are classified into two groups, including FG (phenylalanine-glycine) repeat-containing nucleoporins and scaffold nucleoporins. The FG-repeat domain is composed of multiple FG-rich peptides and is natively unfolded (Denning et al., 2003). FG nucleoporins account for as much as one-third of the molecular mass of the NPC. Since nuclear transport receptor-cargo complexes dock to the NPC by binding to their FG domains, FG nucleoporins are key determinants of nucleocytoplasmic transport. Scaffold nucleoporins form biochemically stable NPC subcomplexes that appear to play important roles as building blocks during NPC biogenesis on the nuclear membrane. NPC core scaffold proteins consist of cage-like structures, which is similar to the structure of coated vesicles (Bonifacino and Glick, 2004; Strambio-De-Castillia et al., 2010). This characteristic similarity suggests that a simple membrane-curving module is the ancient common ancestor of NPCs and coated vesicles (Devos et al., 2006; Alber et al., 2007). In addition to their roles in nucleocytoplasmic transport, NPCs and individual nucleoporins are involved in a large number of cellular processes, including kinetochore and spindle assembly, regulation of gene expression, chromatin organization, and DNA repair (Strambio-De-Castillia et al., 2010; Bukata et al., 2013; Van de Vosse et al., 2013; Table 1).

\section{DEVELOPMENT}

\section{THE ROLE OF EXPORTINS IN PLANT DEVELOPMENT}

The first reported $A$. thaliana importin $\beta$ family mutant was hasty (hst; Telfer and Poethig, 1998). In a forward genetic screen, hst was originally identified as a mutant defective in the transition between developmental phases. The hst mutant exhibits pleiotropic phenotypes, including reduced size of the shoot apical meristem, accelerated vegetative phase change, late flowering under short day conditions, and reduced fertility (Bollman et al., 2003), suggesting that HST is essential for various developmental pathways in plants. The HST gene is the ortholog of H. sapiens exportin 5 (XPO5) and the $S$. cerevisiae bidirectional transporter MSN5 (Bollman et al., 2003). In addition, HST interacts with Ran in yeast two-hybrid assays and localizes to the nuclear periphery (Bollman et al., 2003). Another exportin mutant, paused ( $p s d$ ), was isolated by two different groups (Telfer et al., 1997; Hunter et al., 2003; Li and Chen, 2003). The psd mutant exhibits transiently disrupted organization of the shoot apical meristem, but the timing of the transition to the adult phase of vegetative development is not significantly altered in this mutant (Telfer et al., 1997). PSD encodes an ortholog of exportin-t (XPOT), which mediates nuclear export of tRNAs in yeast (Hellmuth et al., 1998) and human (Kutay et al., 1998).

The different phenotypes observed between the hst and psd exportin mutants can be accounted for by their impaired nuclear export of different cargoes. The hst mutation leads to reduced accumulation of most micro RNAs (miRNAs) but has no effect on the accumulation of tRNAs or endogenous small interfering RNAs (Park et al., 2005). On the contrary, the psd mutation results in compromised tRNA-Tyr processing but does not affect the accumulation or nuclear export of miRNAs (Park etal., 2005). 
Table 1 | Identified plant nucleoporin mutants.

\begin{tabular}{|c|c|c|c|c|}
\hline Nup name & AGI code & Other name & Related pathway & Reference \\
\hline Nup160 & At1g33410 & SAR1 & $\begin{array}{l}\text { Flowering, mRNA export, immunity, cold stress, } \\
\text { and auxin signaling }\end{array}$ & $\begin{array}{l}\text { Dong etal. (2006a), Parry etal. (2006), } \\
\text { Robles et al. (2012), Wiermer et al. (2012) }\end{array}$ \\
\hline Nup96 & At1g80680 & SAR3/MOS3 & $\begin{array}{l}\text { Flowering, mRNA export, immunity, and auxin } \\
\text { signaling }\end{array}$ & Zhang and Li (2005), Parry etal. (2006) \\
\hline TPR/NUA & At1g79280 & & $\begin{array}{l}\text { Flowering, mRNA export, miRNA metabolism, } \\
\text { and auxin signaling }\end{array}$ & Jacob etal. (2007), Xu etal. (2007) \\
\hline Nup58 & & TCU1 & Flowering & Ferrandez-Ayela et al. (2013) \\
\hline Nup62 & & & Flowering & Zhao and Meier (2011) \\
\hline Nup136/Nup1 & & & Flowering and mRNA export & Lu et al. (2010), Tamura etal. (2010) \\
\hline ELYS & & HOS1 & Flowering, mRNA export, and cold stress & $\begin{array}{l}\text { Dong etal. (2006b), Lazaro etal. (2012), } \\
\text { Jung etal. (2013), MacGregor etal. (2013) }\end{array}$ \\
\hline Nup133 & & & Symbiosis & Kanamori et al. (2006), Binder and Parniske (2014) \\
\hline Nup75/85 & & & Symbiosis & Saito et al. (2007), Binder and Parniske (2014) \\
\hline Seh1 & & NENA & Symbiosis, mRNA export and immunity & Groth et al. (2010), Wiermer et al. (2012) \\
\hline Nup88 & & MOS7 & Immunity & Cheng etal. (2009) \\
\hline Nup214 & & LNO1 & Embryo development & Braud et al. (2012) \\
\hline GLE1 & & & Embryo development & Braud etal. (2012) \\
\hline
\end{tabular}

These results clearly indicate that HST and PSD do not share RNA cargoes for nuclear export. Therefore, multiple nuclear export pathways for these small RNAs are required for plant development.

Arabidopsis thaliana has two loci for exportin 1 (XPO1 in vertebrates), designated as XPO1A (At5g17020) and XPO1B (At3g03110); the corresponding proteins share $86 \%$ identity (Blanvillain et al., 2008). Single mutants for XPO1A and XPO1B appear normal, indicating that these genes function redundantly. However, a double mutant homozygote has not been recovered (Blanvillain et al., 2008). Consistent with this observation, cotransmission of mutant alleles of these genes is abolished through the female and is strongly reduced through the male. Female gametophytes of the double mutant show defects ranging from early developmental arrest to disorganized cellular constitution. It was therefore concluded that a maternal copy of XPO1 is required to establish a viable embryo (Blanvillain et al., 2008).

\section{FLOWERING}

As expected, since nucleoporin is involved in fundamental cellular functions, many nucleoporin mutants exhibit pleiotropic phenotypes at both the reproductive and vegetative developmental stages. An early flowering phenotype is commonly observed in various nucleoporin mutants, including nup58 (Ferrandez-Ayela et al., 2013), nup62 (Zhao and Meier, 2011), nup96/sar3/mos3 (Zhang and Li, 2005; Parry et al., 2006), nup136/nup1 (Lu et al., 2010; Tamura et al., 2010), nup160/sar1 (Dong et al., 2006b; Parry et al., 2006), hos1/elys (Ishitani et al., 1998; Lazaro et al., 2012; Jung etal., 2013; MacGregor etal., 2013), and tpr/nua (Jacob etal., 2007; Xu etal., 2007). Xu etal. (2007) found that the tpr/nua mutation affects the expression of flowering-related genes. The expression of floral repressor genes (FLC and MAF4) is reduced in the tpr/nua mutant, while the expression of floral activator genes (FT, SOC, LFY, MYB33, and MYB65) is increased, compared to wild type. Jacob et al. (2007) identified TPR/NUA as a suppressor of FLC expression. The authors also found that tpr/nua flowers earlier than the $f l c$ null mutant, suggesting that FLCdependent and -independent flowering pathways are regulated by TPR/NUA.

The function of HOS1/ELYS in flowering pathway has been well studied. Genetic analysis with hos 1 flc double mutant suggested that the HOS1/ELYS regulates flowering time independent of FLC (Lazaro etal., 2012; Lee etal., 2012a). Moreover, it was found that HOS1/ELYS and ubiquitinated-CONSTANS (CO) physically interact, and HOS1/ELYS regulates CO abundance, particularly during the daylight period (Lazaro et al., 2012). These results suggest that HOS1/ELYS plays a key role in the proteasomedependent degradation of $\mathrm{CO}$ at nuclear pore for control of flowering time (Lazaro et al., 2012; Joon Seo et al., 2013). MacGregor et al. (2013) reported that hos1/elys is affected in circadian clock function, exhibiting a long-period phenotype. Interestingly, Lee etal. (2012b) demonstrated that alternative HOS1 splicing variant is crucial for regulating flowering time, and expresses in a photoperiod-dependent manner.

On the contrary, it was also reported that HOS1/ELYS directly regulates FLC transcription at the chromatin level through interactions with FVE, a histone binding protein (Lee et al., 2012a), and histone deacetylase 6 (HDA6; Jung et al., 2013). FVE and HDA6 interact with each other to function in chromatin silencing in $A$. thaliana (Gu et al., 2011). HOS1/ELYS also interacts with HDA6 and inhibits the binding of HDA6 to FLC chromatin. Moreover, cold treatment induces FLC expression by activating HOS1, which 
inhibits the association of HDA6 with FLC chromatin, resulting in delayed flowering. Therefore, HOS1/ELYS is thought to act as a chromatin-remodeling factor for FLC regulation in response to cold stress. Similar NPC-dependent gene regulation systems that function via chromatin remodeling have been reported in other organisms. In mammalian cells, the nuclear basket component in NPC plays a fundamental role in chromatin organization (Krull et al., 2010). Loss of a nucleoporin induces chromatin condensation all along the nucleoplasmic side of the nuclear envelope, suggesting that NPC maintains pore-associated chromatin in an open state (Grossman et al., 2012). S. cerevisiae Nup170 binds to specific chromatin domains to promote transcriptional repression through its interactions with chromatin-remodeling complex (Van de Vosse et al., 2013). Interactions between chromatin and nucleoporins may represent an initial event in the regulation of gene expression, because this process occurs independently of any preceding transcription (Schmid et al., 2006). Taken together, these results suggest that HOS1/ELYS in NPC also provides a platform for transcriptional regulation to control flowering time in plants.

\section{ESSENTIAL NUCLEOPORINS}

Given their fundamental functions in cellular activities, nucleoporins are thought to be essential for plant viability. However, only a few essential nucleoporins have been identified in $A$. thaliana. A certain allele of nup136/nup1 mutant exhibits gametophytic or embryonic lethal (Lu et al., 2010). A partial loss of Nup88/MOS7 produces variable phenotypes, while a complete loss of Nup88/MOS7 causes lethality (Cheng et al., 2009). This result is consistent with the lethal phenotype of a null nup 88 mutant in D. melanogaster (Uv et al., 2000). Nup214, which provides binding sites for mRNA export factors, was also found to be essential. A mutation in Nup214 abolishes the first asymmetrical cell division during early embryogenesis, resulting in an arrest in embryo development (Braud et al., 2012). Furthermore, a mutation in GLE1, which is thought to work in conjunction with Nup214 in the mRNA export pathway, also leads to embryonic lethality (Braud et al., 2012). In S. cerevisiae, large-scale deletion analysis has already identified many nucleoporins that are essential for cellular viability (Giaever et al., 2002). Further systematic analysis of all $A$. thaliana nucleoporins using T-DNA knockout lines will provide insights into the functional diversity and redundancy of each plant nucleoporin.

\section{HORMONE AND STRESS SIGNALINGS THE ROLE OF IMPORTINS AND EXPORTINS IN HORMONE AND STRESS SIGNALING PATHWAYS}

SAD2 [super sensitive to abscisic acid (ABA) and drought2], which is an ortholog of vertebrate importin 7 and 8, was suggested to regulate various hormone and environmental response pathways in $A$. thaliana. The sad2 mutant exhibits ABA- and stress-hypersensitive induction of luciferase reporter activity (Verslues et al., 2006). Interestingly, sad 2 exhibits ABA hypersensitivity during seed germination and seedling growth, suggesting that SAD2 is involved in nuclear transport of a negative regulator of ABA sensitivity (Verslues et al., 2006). A. thaliana contains one gene (At3g59020) homologous to SAD2 that is in the same clade as importin $7 / 8$
(Figure 1). However, knock out of this gene does not duplicate the sad2 phenotype in response to ABA during seedling growth (Verslues et al., 2006), indicating that SAD2 transports specific cargoes during ABA signaling. In addition to ABA responses, jasmonic acid (JA)-inducible trichome formation is also impaired in the sad2 mutant (Yoshida et al., 2009). The subnuclear localization of the bHLH transcription factor GLABRA3 (GL3), which promotes trichome formation in response to JA, is disrupted in sad2. This suggests that SAD2 regulates (either directly or indirectly) the subnuclear localization of GL3 in response to JA.

The R2R3-type transcription repressor MYB4 is a cargo protein of SAD2 that undergoes nuclear transport (Zhao et al., 2007). MYB4 was previously found to control negative expression of cinnamate 4-hydroxylase (Jin et al., 2000) and, therefore, regulate the synthesis of sinapate esters, which are major photoprotective pigments (Li et al., 1993; Jin et al., 2000). Zhao et al. (2007) demonstrated that sad2 significantly accumulates UV-absorbing pigments and is more tolerant to UV-B irradiation than the wild type. The authors also found that MYB4 coimmunoprecipitates with SAD2 and its nuclear localization requires functional SAD2, suggesting that SAD2 transports MYB4 to the nucleus to help regulate the plant response to UV-B radiation.

Arabidopsis thaliana KPNB1 (At5g53480), which belongs to the IMB1 family, also modulates ABA signaling (Luo et al., 2013). Like the sad 2 mutant, the $k p n b 1$ mutant exhibits increased ABA hypersensitivity during seed germination and cotyledon development. Moreover, the kpnb1 mutation increases stomatal closure in response to $\mathrm{ABA}$, reduces the rate of water loss, and substantially increases drought tolerance. However, KPNB1 was proposed to regulate an ABA pathway independently of SAD2. Loss of function of SAD2 results in early flowering and increased expression of ABA-responsive genes ( $R D 20 A$ and $R A B 18)$ under normal conditions (Verslues et al., 2006). On the contrary, the $k p n b 1$ mutant exhibits late flowering and normal expression of these genes (Luo et al., 2013). These results suggest that KPNB1 transports different cargoes from those of SAD2 to regulate ABA signaling.

As described above, two A. thaliana XPO1 genes (XPO1A, At5g17020 and XPO1B, At3g03110) were previously considered to be functionally redundant (Haasen et al., 1999; Merkle, 2003; Blanvillain etal., 2008). However, the hit2 (heat intolerant 2) mutant, in which a single XPO1A gene is mutated, shows a defect in basal but not acquired thermotolerance (Wu et al., 2010). The hit2 mutant is also sensitive to methyl viologen-induced oxidative stress, and the survival of hit2 seedlings in response to heat stress is affected by light conditions. It was therefore concluded that the hit2 phenotype is attributable to the lack of a sufficient response to heat-induced oxidative injury (Wu et al., 2010). This study clearly indicates that XPO1A has its own specific function and cargoes, which are not necessary for normal growth but are important for plant survival under conditions of sustained or sudden heat stress.

\section{COLD SIGNALING}

There is significant evidence to demonstrate that nucleoporins are required for cold responses. In a screen for altered expression of cold-induced reporter genes, Zhu and colleagues identified several mutants, including nup160/sar1 (Dong et al., 2006b) and hos1/elys (Ishitani et al., 1998; Lee et al., 2001; Dong et al., 2006a). 
The nup160/sar1 mutant impairs cold-responsive gene expression and is sensitive to chilling stress and defective in acquired freezing tolerance (Dong et al., 2006b). On the contrary, hosl/elys exhibits enhanced expression of cold-induced genes in response to low temperature treatment, suggesting that HOS1/ELYS negatively regulates the cold acclimation responses (Lee et al., 2001; MacGregor etal., 2013). Gong et al. (2005) isolated a cryophyte mutant, which exhibits an enhanced cold stress-induction of the master regulator of cold tolerance $(C B F 2)$, and its downstream target genes. The mutant is more tolerant to chilling and freezing stresses but is more sensitive to heat stress. The mutation is found in a DEAD box RNA helicase gene that is identical to the previously identified low expression of osmotically responsive genes 4 (LOS4) locus. The A. thaliana LOS4 is the ortholog of S. cerevisiae DBP5, which interacts with Nup159 and controls mRNA export (discussed below; Kohler and Hurt, 2007). It is demonstrated that LOS4-GFP fusion localizes at the nuclear rim (Gong et al., 2005), suggesting that $A$. thaliana LOS4 interacts with nuclear pore as observed in S. cerevisiae DBP5.

\section{AUXIN HORMONE SIGNALING}

Genetic screening experiments have revealed that nucleoporin is involved in auxin hormone signaling. The auxin resistance1 (axr1) mutant accumulates Aux/IAA proteins, repressors of auxinregulated transcription, resulting in a pleiotropic phenotype consistent with an overall reduction in the auxin response (Lincoln et al., 1990). Two mutant suppressor of axr1 (sar) lines have been isolated and characterized (Cernac et al., 1997; Parry et al., 2006). Parry etal. (2006) found that SAR1 and SAR3 encode Nup160 and Nup96, respectively, which are nucleoporins in the Nup107-Nup160 subcomplex critical for NPC scaffolding. The authors also found that the sarl and sar3 mutants both exhibit impaired nuclear localization of the transcriptional repressor AXR3/INDOLE ACETIC ACID17, suggesting that SAR1 and SAR3 are required for nuclear transport of Aux/IAA proteins in response to auxin signaling. Robles et al. (2012) reported that the nup160/sar1 mutant exhibits an enhanced ethylene response. Mutation of ARF7 or ARF19 almost fully blocks the ethylene hypersensitive phenotype in nup160/sarl, suggesting that auxin signaling is responsible for regulating the magnitude of the ethylene response. Jacob et al. (2007) demonstrated that mutation of TPR/NUA, which forms the nuclear basket of the NPC, also alters the auxin sensitivity of the axr1 mutant to the same level as that of nup96/sar3. Importantly, the levels of several miRNAs, some of which regulate the expression of genes involved in auxin signaling, are significantly reduced in tpr/nua. This observation suggests that NPC may link miRNA metabolism to auxin signaling.

\section{PLANT-PATHOGEN INTERACTION THE ROLE OF TRANSPORTIN-SR IN IMMUNITY}

Serine-arginine rich (SR) proteins are evolutionarily conserved nuclear proteins that play diverse roles in RNA metabolism, including pre-mRNA splicing, non-sense-mediated mRNA decay, and mRNA translation (Reddy, 2004; Long and Caceres, 2009). Transportin-SR (TRN-SR), which belongs to the TNPO3 subfamily (Figure 1A), functions as a nuclear import receptor for
SR proteins in S. cerevisiae (Pemberton et al., 1997) and H. sapiens (Kataoka et al., 1999). Interestingly, knockdown of a TRN-SR gene (TSR-1) in Caenorhabditis elegans results in an early embryonic lethal phenotype, indicating that TRN-SR protein is essential for viability (Longman et al., 2000). A. thaliana has two TRN-SR orthologs (Atlg12930 and At5g62600; Figure 1), one of which was identified as MOS14 (modifier of snc1-1, 14), which plays a key role in immune responses (Xu et al., 2011). The A. thaliana mos14 mutant exhibits impaired nuclear localization of several SR proteins, including mRNA splicing factors (Xu et al., 2011). Consistent with this phenotype, altered splicing patterns of autoactivated Resistance (R) genes (SNC1 and RPS4) were observed in mos14. The authors also found that mRNA splicing of Actin1 and $\beta$-tubulin4 occurs normally in mos14, suggesting there is an mRNA specificity in MOS14-dependent splicing. Taken together, these results suggest that MOS14 is required for plant immunity through proper splicing of R genes. It would be interesting to know whether the other A. thaliana TRN-SR homolog (At1g12930) has different functions and transports different cargoes from MOS14.

\section{THE ROLE OF IMPORTIN $\alpha$ IN Agrobacterium tumefaciens TRANSFORMATION}

To date, two A. thaliana importin $\alpha$ mutants have been reported. Palma et al. (2005) identified the IMPA3 (At4g02150)-deficient mutant mos6 (modifier of snc1, 6), which exhibits partially suppressed constitutive-resistance responses in snc1 mutant. In mos6, salicylic acid (SA) accumulation is inhibited in response to virulent pathogens, suggesting that MOS6 functions upstream of SA biosynthesis in the resistance-signaling pathway. Bhattacharjee et al. (2008) found that four importin $\alpha$ isoforms (IMPA1-4) interact with Agrobacterium tumefaciens virulence proteins (VirD2 and VirE2), which are required for escorting T-DNA into the host's nucleus. However, only the impa4 mutant, but not the other (impa1-3) mutants, exhibits resistance to Agrobacterium tumefaciens transformation. These results suggest that $\alpha 1$ family proteins functionally diverged to acquire specialized roles involving transport of specific cargoes. In further studies, characterization of multiple impa knockout mutants might be required for revealing the functional redundancy of IMPA family in plants.

\section{THE ROLE OF NUCLEOPORINS IN SYMBIOSIS AND IMMUNITY RESPONSES}

Three loss-of-function mutants of Nup133, Nup75/85, and Seh1 have been identified in Lotus japonicus. These nucleoporin mutants are impaired in both fungal (arbuscular mycorrhizal fungi) and bacterial (rhizobium bacteria) symbioses in a temperature-dependent manner (Kanamori etal., 2006; Saito etal., 2007; Groth etal., 2010). It was also found that nup85 nup133 double mutants, but not single mutants, exhibit severe temperature dependent growth and developmental defects (Binder and Parniske, 2014). Although the detailed mechanism of these temperature-dependent phenotypes is unclear, the phenotypes of these mutants are consistent with evidence suggesting temperature-dependent plasticity of nuclear pore plugging in Xenopus laevis (Stoffler et al., 2003). Intracellular root infection 
by either endosymbiont is controlled by the activation of calcium and calmodulin-dependent kinase, a conserved regulatory component of symbiosis signaling. However, these nucleoporin mutants fail to exhibit perinuclear calcium spiking in response to Nod factor, a lipo-chitin oligosaccharide, which leads to differentiation of nodule tissues (Stougaard, 2000). NPC may play a role in calcium spiking by acting as a gate for calcium ions or as a regulator of calcium channels on the nuclear membrane. Although calcium-mediated opening and closing of nuclear baskets has been demonstrated in X. laevis (Stoffler et al., 1999), the molecular mechanism underlying the role of nucleoporin-dependent calcium spiking in the symbiotic process in L. japonicus is unclear.

The A. thaliana snc1 (suppressor of npr1-1, constitutive 1) mutant, which contains a gain-of-function mutation in the $\mathrm{R}$ (resistance) gene, shows constitutive activation of diseaseresistance responses against pathogens. In a screen for suppressors of snc1, Li and colleagues identified several mos (modifier of snc1) mutants, including nup96/sar3/mos3 (Zhang and Li, 2005) and nup88/mos7 (Cheng et al., 2009). Both mos mutants have abolished SA accumulation, pathogenesis-related (PR) gene expression, and basal and R-gene-mediated resistance. Importantly, in nup88/mos7, nuclear accumulation of sncl and the defense signaling components NPR1 (non-expresser of PR genes 1) and EDS1 (enhanced disease susceptibility 1) is significantly reduced, while nuclear retention of other tested proteins is unaffected (Cheng et al., 2009). This result suggests that cargo specificity between nuclear proteins and Nup88 is critical for immune responses. Nup96 is a component of the Nup107Nup160 subcomplex, which is the evolutionarily conserved and largest subcomplex in the NPC. Wiermer et al. (2012) also examined whether other Nup107-Nup160 subcomplex members are involved in immunity. Among eight putative complex members examined, only plants with defects in Nup96, Nup160, or Seh1 are impaired in basal resistance and exhibit a suppressed auto-immunity phenotype in sncl (Wiermer et al., 2012). The nup160/sar1 and seh1 mutants also exhibit compromised accumulation of EDS1, an essential regulator of basal resistance, as observed in nup88/mos7 plants. These results suggest that the functions of these nucleoporins partially overlap, but nucleoporins also play specific regulatory roles in plant immune responses.

\section{RNA METABOLISM REGULATION OF mIRNA ACTIVITY BY IMPORTIN}

A forward genetic screen for miRNA activity led to the identification of the emal (enhanced miRNA activityl) mutant, which is allelic to sad2 (Wang et al., 2011). Wang etal. (2011) found that the levels of endogenous miRNA targets (SPL3, MYB54, and $C U C 2$ ) were reduced by $40-50 \%$ in sad2/ema1, indicating that SAD2/EMA1 is involved in general miRNA production. The authors also demonstrated that the miRNA effector complexes in emal contain higher amounts of miRNAs and elevated mRNA cleavage activity compared to the wild type, indicating that EMA1 modulates miRNA activity by influencing the loading of miRNAs into ARGONAUTE1 (AGO1) complexes. On the contrary, emal has no effect on the accumulation of miRNAs or AGO1 on their cytoplasmic or nuclear distribution. These results suggest that SAD2/EMA1 functions as a negative regulator of the miRNA pathway. Although it is unclear whether SAD2/EMA1 directly or indirectly interacts with AGO1 complexes, SAD2/EMA1 may sequester excessive miRNAs to prevent their loading into the AGO1 complex. Alternatively, nuclear-cytoplasmic distribution of SAD2/EMA1 cargoes may be important for the regulation of miRNA activity.

\section{REGULATION OF mRNA EXPORT AT NUCLEAR PORE}

The mRNA-transport receptor complex physically interacts with the FG-repeats of FG nucleoporins, which allow it to overcome the permeability barrier of the NPC (Kohler and Hurt, 2007). An aberrant mRNA export phenotype is widely observed in nucleoporin mutants, including nup96/sar3/mos3 (Zhang and Li, 2005; Parry et al., 2006), nup136/nup1 (Lu et al., 2010; Tamura et al., 2010), nup160/sar1 (Dong et al., 2006b; Parry et al., 2006), hos1/elys (MacGregor et al., 2013), seh1 (Wiermer et al., 2012), and tpr/nua (Jacob et al., 2007; Xu etal., 2007). In addition, deficiencies of NPCassociated factors also result in abnormal mRNA export. Mutation in LOS4, whose yeast ortholog is known to interact with nuclear pore and regulate mRNA export (Kohler and Hurt, 2007), results in abnormal mRNAs accumulation in nucleus (Gong et al., 2005). Lu et al. (2010) reported that TREX-2 complex, which is anchored by Nup136/Nup1, is required for mRNA export in A. thaliana. Although several components involved in the mRNA export have been isolated, further study will be required for understanding the molecular mechanisms and physiological roles of mRNA export system in plants.

\section{CONCLUDING REMARKS}

As discussed above, many nucleocytoplasmic transport mutants exhibit pleiotropic phenotypes. MacGregor et al. (2013) demonstrated that los 4 and several nucleoporin mutants including nup160/sar1, nup107, nua, and hos1/elys all exhibit long-period circadian phenotypes and alterations to clock gene expression. Since many of the signaling pathways are under control of circadian clock, it is raised possibility that impairing in nucleocytoplasmic transport leads indirect effects on the signaling pathways. Moreover, tpr/nua was found to increase largely in the amount of mRNA compared with wild type, leading to broad-scale transcriptome alterations (Jacob et al., 2007). This result also suggests that the nucleocytoplasmic transport has indirect effect on the signaling. Further studies are needed in order to differentiate between direct and indirect role of nucleocytoplasmic transport in plants.

Recent years have seen major progress in our understanding of the molecular mechanisms underlying nucleocytoplasmic transport in plant cells. It is now firmly established that these transport systems are responsible for developmental and signaling pathways that are indispensable for plant growth. Although nucleocytoplasmic transport is an evolutionarily conserved system in eukaryotic cells, plants appear to have developed functionally divergent cargoes and regulatory mechanisms, especially in response to environmental signaling. Achieving a deeper understanding of the mechanisms by which nuclear transport receptors exert these functions will require the identification of the 
cargo molecules that are transported by these nuclear transport receptors.

\section{ACKNOWLEDGMENTS}

This work was supported by a Grant-in-Aid for Scientific Research to Kentaro Tamura (no. 25650096) and a Specially Promoted Research Grant-in-Aid for Scientific Research to Ikuko HaraNishimura (no. 22000014) from the Japan Society for the Promotion of Science.

\section{REFERENCES}

Alber, F., Dokudovskaya, S., Veenhoff, L. M., Zhang, W., Kipper, J., Devos, D., et al. (2007). The molecular architecture of the nuclear pore complex. Nature 450, 695-701. doi: 10.1038/nature06405

Bednenko, J., Cingolani, G., and Gerace, L. (2003). Nucleocytoplasmic transport: navigating the channel. Traffic 4, 127-135. doi: 10.1034/j.1600-0854.2003. 00109.x

Bhattacharjee, S., Lee, L. Y., Oltmanns, H., Cao, H., Veena, Cuperus, J., et al. (2008). IMPa-4, an Arabidopsis importin alpha isoform, is preferentially involved in Agrobacterium-mediated plant transformation. Plant Cell 20, 2661-2680. doi: 10.1105/tpc.108.060467

Binder, A., and Parniske, M. (2014). Analysis of the Lotus japonicus nuclear pore NUP107-160 subcomplex reveals pronounced structural plasticity and functional redundancy. Front. Plant Sci. 4:552. doi: 10.3389/fpls.2013.00552

Blanvillain, R., Boavida, L. C., Mccormick, S., and Ow, D. W. (2008). Exportin 1 genes are essential for development and function of the gametophytes in Arabidopsis thaliana. Genetics 180, 1493-1500. doi: 10.1534/genetics.108.094896

Bollman, K. M., Aukerman, M. J., Park, M. Y., Hunter, C., Berardini, T. Z., and Poethig, R. S. (2003). HASTY, the Arabidopsis ortholog of exportin 5/MSN5 regulates phase change and morphogenesis. Development 130, 1493-1504. doi: 10.1242/dev.00362

Bonifacino, J. S., and Glick, B. S. (2004). The mechanisms of vesicle budding and fusion. Cell 116, 153-166. doi: 10.1016/S0092-8674(03)01079-1

Braud, C., Zheng, W., and Xiao, W. (2012). LONO1 encoding a nucleoporin is required for embryogenesis and seed viability in Arabidopsis. Plant Physiol. 160, 823-836. doi: 10.1104/pp.112.202192

Bukata, L., Parker, S. L., and D'Angelo, M. A. (2013). Nuclear pore complexes in the maintenance of genome integrity. Curr. Opin. Cell Biol. 25, 378-386. doi: 10.1016/j.ceb.2013.03.002

Cernac, A., Lincoln, C., Lammer, D., and Estelle, M. (1997). The SAR1 gene of Arabidopsis acts downstream of the AXR1 gene in auxin response. Development 124, 1583-1591.

Chang, C. W., Counago, R. L., Williams, S. J., Boden, M., and Kobe, B. (2012). Crystal structure of rice importin-alpha and structural basis of its interaction with plant-specific nuclear localization signals. Plant Cell 24, 5074-5088. doi: 10.1105/tpc.112.104422

Cheng, Y. T., Germain, H., Wiermer, M., Bi, D., Xu, F., Garcia, A. V., et al. (2009). Nuclear pore complex component MOS7/Nup88 is required for innate immunity and nuclear accumulation of defense regulators in Arabidopsis. Plant Cell 21, 2503-2516. doi: 10.1105/tpc.108.064519

Cingolani, G., Bednenko, J., Gillespie, M. T., and Gerace, L. (2002). Molecular basis for the recognition of a nonclassical nuclear localization signal by importin beta. Mol. Cell 10, 1345-1353. doi: 10.1016/S1097-2765(02)00727-X

Cingolani, G., Petosa, C., Weis, K., and Muller, C. W. (1999). Structure of importinbeta bound to the IBB domain of importin-alpha. Nature 399, 221-229. doi: $10.1038 / 20367$

Conti, E., Uy, M., Leighton, L., Blobel, G., and Kuriyan, J. (1998). Crystallographic analysis of the recognition of a nuclear localization signal by the nuclear import factor karyopherin alpha. Cell 94, 193-204. doi: 10.1016/S0092-8674(00)81419-1

Cronshaw, J. M., Krutchinsky, A. N., Zhang, W., Chait, B. T., and Matunis, M. J. (2002). Proteomic analysis of the mammalian nuclear pore complex. J. Cell Biol. 158, 915-927. doi: 10.1083/jcb.200206106

Denning, D. P., Patel, S. S., Uversky, V., Fink, A. L., and Rexach, M. (2003) Disorder in the nuclear pore complex: the FG repeat regions of nucleoporins are natively unfolded. Proc. Natl. Acad. Sci. U.S.A. 100, 2450-2455. doi: 10.1073/pnas.0437902100
Devos, D., Dokudovskaya, S., Williams, R., Alber, F., Eswar, N., Chait, B. T., et al. (2006). Simple fold composition and modular architecture of the nuclear pore complex. Proc. Natl. Acad. Sci. U.S.A. 103, 2172-2177. doi: 10.1073/pnas.0506345103

Dong, C.-H., Agarwal, M., Zhang, Y., Xie, Q., and Zhu, J.-K. (2006a). The negative regulator of plant cold responses, HOS1, is a RING E3 ligase that mediates the ubiquitination and degradation of ICE1. Proc. Natl. Acad. Sci. U.S.A. 103, 82818286. doi: 10.1073/pnas.0602874103

Dong, C.-H., Hu, X., Tang, W., Zheng, X., Kim, Y. S., Lee, B.-H., et al. (2006b) A putative Arabidopsis nucleoporin, AtNUP160, is critical for RNA export and required for plant tolerance to cold stress. Mol. Cell. Biol. 26, 9533-9543. doi: 10.1128/MCB.01063-06

Ferrandez-Ayela, A., Alonso-Peral, M. M., Sanchez-Garcia, A. B., Micol-Ponce, R., Perez-Perez, J. M., Micol, J. L., et al. (2013). Arabidopsis TRANSCURVATA1 encodes NUP58, a component of the nucleopore central channel. PLoS ONE 8:e67661. doi: 10.1371/journal.pone.0067661

Giaever, G., Chu, A. M., Ni, L., Connelly, C., Riles, L., Veronneau, S., et al. (2002). Functional profiling of the Saccharomyces cerevisiae genome. Nature 418, 387391. doi: $10.1038 /$ nature00935

Goldfarb, D. S., Corbett, A. H., Mason, D. A., Harreman, M. T., and Adam, S. A. (2004). Importin alpha: a multipurpose nuclear-transport receptor. Trends Cell Biol. 14, 505-514. doi: 10.1016/j.tcb.2004.07.016

Gong, Z., Dong, C. H., Lee, H., Zhu, J., Xiong, L., Gong, D., et al. (2005). A DEAD box RNA helicase is essential for mRNA export and important for development and stress responses in Arabidopsis. Plant Cell 17, 256-267. doi: $10.1105 /$ tpc. 104.027557

Grossman, E., Medalia, O., and Zwerger, M. (2012). Functional architecture of the nuclear pore complex. Annu. Rev. Biophys. 41, 557-584. doi: 10.1146/annurevbiophys-050511-102328

Groth, M., Takeda, N., Perry, J., Uchida, H., Draxl, S., Brachmann, A., et al. (2010). NENA, a Lotus japonicus homolog of Sec13, is required for rhizodermal infection by arbuscular mycorrhiza fungi and rhizobia but dispensable for cortical endosymbiotic development. Plant Cell 22, 2509-2526. doi: 10.1105/tpc.109.069807

Gu, X., Jiang, D., Yang, W., Jacob, Y., Michaels, S. D., and He, Y. (2011). Arabidopsis homologs of retinoblastoma-associated protein 46/48 associate with a histone deacetylase to act redundantly in chromatin silencing. PLoS Genet. 7:e1002366. doi: 10.1371/journal.pgen.1002366

Haasen, D., Kohler, C., Neuhaus, G., and Merkle, T. (1999). Nuclear export of proteins in plants: AtXPO1 is the export receptor for leucine-rich nuclear export signals in Arabidopsis thaliana. Plant J. 20, 695-705. doi: 10.1046/j.1365313X.1999.00644.x

Haasen, D., and Merkle, T. (2002). Characterization of an Arabidopsis thaliana homologue of the nuclear export receptor CAS by its interaction with Importin alpha. Plant Biol. 4, 432-439. doi: 10.1055/s-2002-34124

Hellmuth, K., Lau, D. M., Bischoff, F. R., Kunzler, M., Hurt, E., and Simos, G. (1998). Yeast Los $1 p$ has properties of an exportin-like nucleocytoplasmic transport factor for tRNA. Mol. Cell. Biol. 18, 6374-6386.

Hunter, C. A., Aukerman, M. J., Sun, H., Fokina, M., and Poethig, R. S. (2003). PAUSED encodes the Arabidopsis exportin-t ortholog. Plant Physiol. 132, 21352143. doi: 10.1104/pp.103.023309

Ishitani, M., Xiong, L., Lee, H., Stevenson, B., and Zhu, J. K. (1998). HOS1, a genetic locus involved in cold-responsive gene expression in Arabidopsis. Plant Cell 10, 1151-1161.

Jacob, Y., Mongkolsiriwatana, C., Veley, K. M., Kim, S. Y., and Michaels, S. D. (2007). The nuclear pore protein AtTPR is required for RNA homeostasis, flowering time, and auxin signaling. Plant Physiol. 144, 1383-1390. doi: 10.1104/pp.107.100735

Jin, H., Cominelli, E., Bailey, P., Parr, A., Mehrtens, F., Jones, J., et al. (2000). Transcriptional repression by AtMYB4 controls production of UV-protecting sunscreens in Arabidopsis. EMBO J. 19, 6150-6161. doi: 10.1093/emboj/19.22.6150

Joon Seo, P., Jung, J. H., Park, M. J., Lee, K., and Park, C. M. (2013). Controlled turnover of CONSTANS protein by the HOS1 E3 ligase regulates floral transition at low temperatures. Plant Signal. Behav. 8:e23780. doi: 10.4161/psb.23780

Jung, J. H., Park, J. H., Lee, S., To, T. K., Kim, J. M., Seki, M., et al. (2013). The cold signaling attenuator HIGH EXPRESSION OF OSMOTICALLY RESPONSIVE GENE1 activates FLOWERING LOCUS C transcription via chromatin remodeling under short-term cold stress in Arabidopsis. Plant Cell 25, 4378-4390. doi: $10.1105 /$ tpc. 113.118364 
Kanamori, N., Madsen, L. H., Radutoiu, S., Frantescu, M., Quistgaard, E. M., Miwa, H., et al. (2006). A nucleoporin is required for induction of $\mathrm{Ca}^{2+}$ spiking in legume nodule development and essential for rhizobial and fungal symbiosis. Proc. Natl. Acad. Sci. U.S.A. 103, 359-364. doi: 10.1073/pnas.0508883103

Kataoka, N., Bachorik, J. L., and Dreyfuss, G. (1999). Transportin-SR, a nuclear import receptor for SR proteins. J. Cell Biol. 145, 1145-1152. doi: $10.1083 /$ jcb.145.6.1145

Kohler, A., and Hurt, E. (2007). Exporting RNA from the nucleus to the cytoplasm. Nat. Rev. Mol. Cell Biol. 8, 761-773. doi: 10.1038/nrm2255

Kosugi, S., Hasebe, M., Matsumura, N., Takashima, H., Miyamoto-Sato, E., Tomita, M., et al. (2009). Six classes of nuclear localization signals specific to different binding grooves of importin alpha. J. Biol. Chem. 284, 478-485. doi: 10.1074/jbc.M807017200

Krull, S., Dorries, J., Boysen, B., Reidenbach, S., Magnius, L., Norder, H., et al. (2010). Protein Tpr is required for establishing nuclear pore-associated zones of heterochromatin exclusion. EMBO J. 29, 1659-1673. doi: 10.1038/emboj.2010.54

Kutay, U., Lipowsky, G., Izaurralde, E., Bischoff, F. R., Schwarzmaier, P., Hartmann, E., et al. (1998). Identification of a tRNA-specific nuclear export receptor. Mol. Cell 1, 359-369. doi: 10.1016/S1097-2765(00)80036-2

Lazaro, A., Valverde, F., Pineiro, M., and Jarillo, J. A. (2012). The Arabidopsis E3 ubiquitin ligase HOS1 negatively regulates CONSTANS abundance in the photoperiodic control of flowering. Plant Cell 24, 982-999. doi: $10.1105 /$ tpc. 110.081885

Lee, H., Xiong, L., Gong, Z., Ishitani, M., Stevenson, B., and Zhu, J. K. (2001). The Arabidopsis HOS1 gene negatively regulates cold signal transduction and encodes a RING finger protein that displays cold-regulated nucleo-cytoplasmic partitioning. Genes Dev. 15, 912-924. doi: 10.1101/gad.866801

Lee, J. H., Kim, J. J., Kim, S. H., Cho, H. J., Kim, J., and Ahn, J. H. (2012a). The E3 ubiquitin ligase HOS1 regulates low ambient temperature-responsive flowering in Arabidopsis thaliana. Plant Cell Physiol. 53, 1802-1814. doi: 10.1093/pcp/pcs123

Lee, J. H., Kim, S. H., Kim, J. J., and Ahn, J. H. (2012b). Alternative splicing and expression analysis of High expression of osmotically responsive genes1 (HOS1) in Arabidopsis. BMB Rep. 45, 515-520. doi: 10.5483/BMBRep.2012.45.9.092

Lee, S. J., Sekimoto, T., Yamashita, E., Nagoshi, E., Nakagawa, A., Imamoto, N., et al. (2003). The structure of importin-beta bound to SREBP-2: nuclear import of a transcription factor. Science 302, 1571-1575. doi: 10.1126/science.1088372

Li, J., and Chen, X. (2003). PAUSED, a putative exportin-t, acts pleiotropically in Arabidopsis development but is dispensable for viability. Plant Physiol. 132, 1913-1924. doi: 10.1104/pp.103.023291

Li, J., Ou-Lee, T. M., Raba, R., Amundson, R. G., and Last, R. L. (1993). Arabidopsis flavonoid mutants are hypersensitive to UV-B irradiation. Plant Cell 5, 171-179. doi: 10.1105/tpc.5.2.171

Lincoln, C., Britton, J. H., and Estelle, M. (1990). Growth and development of the axr1 mutants of Arabidopsis. Plant Cell 2, 1071-1080. doi: 10.1105/tpc.2.11.1071

Long, J. C., and Caceres, J. F. (2009). The SR protein family of splicing factors: master regulators of gene expression. Biochem. J. 417, 15-27. doi: 10.1042/BJ20081501

Longman, D., Johnstone, I. L., and Caceres, J. F. (2000). Functional characterization of SR and SR-related genes in Caenorhabditis elegans. EMBO J. 19, 1625-1637. doi: 10.1093/emboj/19.7.1625

Lott, K., and Cingolani, G. (2011). The importin beta binding domain as a master regulator of nucleocytoplasmic transport. Biochim. Biophys. Acta 1813, 15781592. doi: 10.1016/j.bbamcr.2010.10.012

Lu, Q., Tang, X., Tian, G., Wang, F., Liu, K., Nguyen, V., et al. (2010). Arabidopsis homolog of the yeast TREX-2 mRNA export complex: components and anchoring nucleoporin. Plant J. 61, 259-270. doi: 10.1111/j.1365-313X.2009.04048.x

Luo, Y., Wang, Z., Ji, H., Fang, H., Wang, S., Tian, L., et al. (2013). An Arabidopsis homolog of importin betal is required for ABA response and drought tolerance. Plant J. 75, 377-389. doi: 10.1111/tpj.12207

MacGregor, D. R., Gould, P., Foreman, J., Griffiths, J., Bird, S., Page, R., et al. (2013). HIGH EXPRESSION OF OSMOTICALLY RESPONSIVE GENES1 is required for circadian periodicity through the promotion of nucleo-cytoplasmic mRNA export in Arabidopsis. Plant Cell 25, 4391-4404. doi: 10.1105/tpc.113.114959

Marfori, M., Mynott, A., Ellis, J. J., Mehdi, A. M., Saunders, N. F., Curmi, P. M., et al. (2011). Molecular basis for specificity of nuclear import and prediction of nuclear localization. Biochim. Biophys. Acta 1813, 1562-1577. doi: 10.1016/j.bbamcr.2010.10.013

Mason, D. A., Fleming, R. J., and Goldfarb, D. S. (2002). Drosophila melanogaster importin alpha1 and alpha3 can replace importin alpha2 during spermatogenesis but not oogenesis. Genetics 161, 157-170.
Mason, D. A., Stage, D. E., and Goldfarb, D. S. (2009). Evolution of the metazoan-specific importin alpha gene family. J. Mol. Evol. 68, 351-365. doi: $10.1007 /$ s00239-009-9215-8

Meier, I., and Somers, D. E. (2011). Regulation of nucleocytoplasmic trafficking in plants. Curr. Opin. Plant Biol. 14, 538-546. doi: 10.1016/j.pbi.2011. 06.005

Merkle, T. (2003). Nucleo-cytoplasmic partitioning of proteins in plants: implications for the regulation of environmental and developmental signalling. Curr. Genet. 44, 231-260. doi: 10.1007/s00294-003-0444-x

Merkle, T. (2011). Nucleo-cytoplasmic transport of proteins and RNA in plants. Plant Cell Rep. 30, 153-176. doi: 10.1007/s00299-010-0928-3

Mosammaparast, N., and Pemberton, L. F. (2004). Karyopherins: from nucleartransport mediators to nuclear-function regulators. Trends Cell Biol. 14, 547-556. doi: 10.1016/j.tcb.2004.09.004

O'Reilly, A. J., Dacks, J. B., and Field, M. C. (2011). Evolution of the karyopherinbeta family of nucleocytoplasmic transport factors; ancient origins and continued specialization. PLoS ONE 6:e19308. doi: 10.1371/journal.pone.0019308

Palma, K., Zhang, Y., and Li, X. (2005). An importin alpha homolog, MOS6, plays an important role in plant innate immunity. Curr. Biol. 15, 1129-1135. doi: 10.1016/j.cub.2005.05.022

Park, M. Y., Wu, G., Gonzalez-Sulser, A., Vaucheret, H., and Poethig, R. S. (2005). Nuclear processing and export of microRNAs in Arabidopsis. Proc. Natl. Acad. Sci. U.S.A. 102, 3691-3696. doi: 10.1073/pnas.0405570102

Parry, G. (2013). Assessing the function of the plant nuclear pore complex and the search for specificity. J. Exp. Bot. 64, 833-845. doi: 10.1093/jxb/ers289

Parry, G., Ward, S., Cernac, A., Dharmasiri, S., and Estelle, M. (2006). The Arabidopsis SUPPRESSOR OF AUXIN RESISTANCE proteins are nucleoporins with an important role in hormone signaling and development. Plant Cell 18, 1590-1603. doi: 10.1105/tpc.106.041566

Pemberton, L. F., Rosenblum, J. S., and Blobel, G. (1997). A distinct and parallel pathway for the nuclear import of an mRNA-binding protein. J. Cell Biol. 139, 1645-1653. doi: 10.1083/jcb.139.7.1645

Raices, M., and D'Angelo, M. A. (2012). Nuclear pore complex composition: a new regulator of tissue-specific and developmental functions. Nat. Rev. Mol. Cell Biol. 13, 687-699. doi: 10.1038/nrm3461

Reddy, A. S. (2004). Plant serine/arginine-rich proteins and their role in pre-mRNA splicing. Trends Plant Sci. 9, 541-547. doi: 10.1016/j.tplants.2004.09.007

Robles, L. M., Deslauriers, S. D., Alvarez, A. A., and Larsen, P. B. (2012). A lossof-function mutation in the nucleoporin AtNUP160 indicates that normal auxin signalling is required for a proper ethylene response in Arabidopsis. J. Exp. Bot. 63, 2231-2241. doi: 10.1093/jxb/err424

Rout, M. P., Aitchison, J. D., Suprapto, A., Hjertaas, K., Zhao, Y., and Chait, B. T. (2000). The yeast nuclear pore complex: composition, architecture, and transport mechanism. J. Cell Biol. 148, 635-651. doi: 10.1083/jcb.148.4.635

Saito, K., Yoshikawa, M., Yano, K., Miwa, H., Uchida, H., Asamizu, E., et al. (2007). NUCLEOPORIN85 is required for calcium spiking, fungal and bacterial symbioses, and seed production in Lotus japonicus. Plant Cell 19, 610-624. doi: 10.1105/tpc. 106.046938

Schmid, M., Arib, G., Laemmli, C., Nishikawa, J., Durussel, T., and Laemmli, U. K. (2006). Nup-PI: the nucleopore-promoter interaction of genes in yeast. Mol. Cell 21, 379-391. doi: 10.1016/j.molcel.2005.12.012

Smith, H. M., Hicks, G. R., and Raikhel, N. V. (1997). Importin alpha from Arabidopsis thaliana is a nuclear import receptor that recognizes three classes of import signals. Plant Physiol. 114, 411-417. doi: 10.1104/pp.114.2.411

Stewart, M. (2007). Molecular mechanism of the nuclear protein import cycle. Nat. Rev. Mol. Cell Biol. 8, 195-208. doi: 10.1038/nrm2114

Stoffler, D., Feja, B., Fahrenkrog, B., Walz, J., Typke, D., and Aebi, U. (2003). Cryo-electron tomography provides novel insights into nuclear pore architecture: implications for nucleocytoplasmic transport. J. Mol. Biol. 328, 119-130. doi: 10.1016/S0022-2836(03)00266-3

Stoffler, D., Goldie, K. N., Feja, B., and Aebi, U. (1999). Calcium-mediated structural changes of native nuclear pore complexes monitored by time-lapse atomic force microscopy. J. Mol. Biol. 287, 741-752. doi: 10.1006/jmbi.1999.2637

Stougaard, J. (2000). Regulators and regulation of legume root nodule development. Plant Physiol. 124, 531-540. doi: 10.1104/pp.124.2.531

Strambio-De-Castillia, C., Niepel, M., and Rout, M. P. (2010). The nuclear pore complex: bridging nuclear transport and gene regulation. Nat. Rev. Mol. Cell Biol. 11, 490-501. doi: 10.1038/nrm2928 
Strom, A. C., and Weis, K. (2001). Importin-beta-like nuclear transport receptors. Genome Biol. 2, REVIEWS3008. doi: 10.1186/gb-2001-2-6-reviews3008

Tamura, K., Fukao, Y., Iwamoto, M., Haraguchi, T., and Hara-Nishimura, I. (2010). Identification and characterization of nuclear pore complex components in Arabidopsis thaliana. Plant Cell 22, 4084-4097. doi: 10.1105/tpc.110.079947

Tamura, K., and Hara-Nishimura, I. (2013). The molecular architecture of the plant nuclear pore complex. J. Exp. Bot. 64, 823-832. doi: 10.1093/jxb/ers258

Telfer, A., Bollman, K. M., and Poethig, R. S. (1997). Phase change and the regulation of trichome distribution in Arabidopsis thaliana. Development 124, 645-654.

Telfer, A., and Poethig, R. S. (1998). HASTY: a gene that regulates the timing of shoot maturation in Arabidopsis thaliana. Development 125, 1889-1898.

Uv, A. E., Roth, P., Xylourgidis, N., Wickberg, A., Cantera, R., and Samakovlis, C. (2000). members only encodes a Drosophila nucleoporin required for rel protein import and immune response activation. Genes Dev. 14, 1945-1957.

Van de Vosse, D. W., Wan, Y., Lapetina, D. L., Chen, W. M., Chiang, J. H., Aitchison, J. D., et al. (2013). A role for the nucleoporin Nup170p in chromatin structure and gene silencing. Cell 152, 969-983. doi: 10.1016/j.cell.2013.01.049

Verslues, P. E., Guo, Y., Dong, C. H., Ma, W., and Zhu, J. K. (2006). Mutation of SAD2, an importin beta-domain protein in Arabidopsis, alters abscisic acid sensitivity. Plant J. 47, 776-787. doi: 10.1111/j.1365-313X.2006.02833.x

Wang, W., Ye, R., Xin, Y., Fang, X., Li, C., Shi, H., et al. (2011). An importin beta protein negatively regulates microRNA activity in Arabidopsis. Plant Cell 23 , 3565-3576. doi: 10.1105/tpc.111.091058

Wiermer, M., Cheng, Y. T., Imkampe, J., Li, M., Wang, D., Lipka, V., et al. (2012). Putative members of the Arabidopsis Nup107-160 nuclear pore sub-complex contribute to pathogen defense. Plant J. 70, 796-808. doi: 10.1111/j.1365313X.2012.04928.x

Wu, S. J., Wang, L. C., Yeh, C. H., and Lu, C. A. (2010). Isolation and characterization of the Arabidopsis heat-intolerant 2 (hit2) mutant reveal the essential role of the nuclear export receptor EXPORTIN1A (XPO1A) in plant heat tolerance. New Phytol. 186, 833-842. doi: 10.1111/j.1469-8137.2010.03225.x

$\mathrm{Xu}$, D., Farmer, A., and Chook, Y. M. (2010). Recognition of nuclear targeting signals by Karyopherin-beta proteins. Curr. Opin. Struct. Biol. 20, 782-790. doi: 10.1016/j.sbi.2010.09.008

Xu, S., Zhang, Z., Jing, B., Gannon, P., Ding, J., Xu, F., et al. (2011). Transportin-SR is required for proper splicing of resistance genes and plant immunity. PLoS Genet. 7:e1002159. doi: 10.1371/journal.pgen.1002159
Xu, X. M., Rose, A., Muthuswamy, S., Jeong, S. Y., Venkatakrishnan, S., Zhao, Q., et al. (2007). NUCLEAR PORE ANCHOR, the Arabidopsis homolog of Tpr/Mlp1/Mlp2/megator, is involved in mRNA export and SUMO homeostasis and affects diverse aspects of plant development. Plant Cell 19, 1537-1548. doi: $10.1105 /$ tpc.106.049239

Yasuhara, N., Oka, M., and Yoneda, Y. (2009). The role of the nuclear transport system in cell differentiation. Semin. Cell Dev. Biol. 20, 590-599. doi: 10.1016/j.semcdb.2009.05.003

Yoshida, Y., Sano, R., Wada, T., Takabayashi, J., and Okada, K. (2009). Jasmonic acid control of GLABRA3 links inducible defense and trichome patterning in Arabidopsis. Development 136, 1039-1048. doi: 10.1242/dev.030585

Zhang, Y., and Li, X. (2005). A putative nucleoporin 96 Is required for both basal defense and constitutive resistance responses mediated by suppressor of npr1-1, constitutive 1. Plant Cell 17, 1306-1316. doi: 10.1105/tpc.104.029926

Zhao, J., Zhang, W., Zhao, Y., Gong, X., Guo, L., Zhu, G., et al. (2007). SAD2, an importin-like protein, is required for UV-B response in Arabidopsis by mediating MYB4 nuclear trafficking. Plant Cell 19, 3805-3818. doi: 10.1105/tpc.106.048900 Zhao, Q., and Meier, I. (2011). Identification and characterization of the Arabidopsis FG-repeat nucleoporin Nup62. Plant Signal. Behav. 6, 330-334. doi: $10.4161 /$ psb.6.3.13402

Conflict of Interest Statement: The authors declare that the research was conducted in the absence of any commercial or financial relationships that could be construed as a potential conflict of interest.

Received: 12 February 2014; paper pending published: 21 February 2014; accepted: 12 March 2014; published online: 02 April 2014.

Citation: Tamura K and Hara-Nishimura I (2014) Functional insights of nucleocytoplasmic transport in plants. Front. Plant Sci. 5:118. doi: 10.3389/fpls.2014.00118

This article was submitted to Plant Cell Biology, a section of the journal Frontiers in Plant Science.

Copyright (C) 2014 Tamura and Hara-Nishimura. This is an open-access article distributed under the terms of the Creative Commons Attribution License (CC BY). The use, distribution or reproduction in other forums is permitted, provided the original author(s) or licensor are credited and that the original publication in this journal is cited, in accordance with accepted academic practice. No use, distribution or reproduction is permitted which does not comply with these terms. 